\title{
Belgeo
}

Revue belge de géographie

\section{Exploration touristique des rivières de France, entre description et mythification (1904-1924)}

Tourist exploration of French rivers, between description and mythologizing (1904-1924)

\section{Eric Levet-Labry}

\section{(2)enEdition \\ Journals}

Édition électronique

URL : http://journals.openedition.org/belgeo/7151

DOI : 10.4000/belgeo.7151

ISSN : 2294-9135

\section{Éditeur :}

National Committee of Geography of Belgium, Société Royale Belge de Géographie

\section{Référence électronique}

Eric Levet-Labry, «Exploration touristique des rivières de France, entre description et mythification (1904-1924) », Belgeo [En ligne], 3 | 2012, mis en ligne le 13 mars 2013, consulté le 19 avril 2019. URL: http://journals.openedition.org/belgeo/7151; DOI : 10.4000/belgeo.7151

Ce document a été généré automatiquement le 19 avril 2019

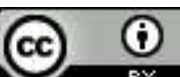

Belgeo est mis à disposition selon les termes de la licence Creative Commons Attribution 4.0 International. 


\section{Exploration touristique des rivières de France, entre description et mythification (1904-1924)}

Tourist exploration of French rivers, between description and mythologizing

(1904-1924)

Eric Levet-Labry

1 Les déplacements touristiques constituent à l'aube $\mathrm{du} \mathrm{XX}^{\mathrm{e}}$ siècle une activité qui, après avoir été réservée à l'aristocratie, se diffuse dans les classes bourgeoises (Boyer, 2002). Avec la popularisation des moyens de transport et leur multiplicité, en peu de temps on passe des voitures attelées, au rail, à la bicyclette, puis à l'automobile (Bertho-Lavenir, 1999). Cela engendre une démocratisation du voyage et le développement du tourisme. Ce tourisme est aujourd'hui communément entendu comme un déplacement hors de son lieu de résidence habituel, nécessitant un hébergement, dans un but de loisirs, un but professionnel (tourisme d'affaires) ou un but sanitaire (tourisme de santé). Cette définition de l'Organisation Mondiale du Tourisme, si elle permet une homogénéisation des données à des fins statistiques, reste trop générale et limitée. Le tourisme envisagé comme "un système d'acteurs, de pratiques et de lieux qui a pour finalité de permettre aux individus de se déplacer dans leur récréation hors de leurs lieux de vie habituels afin d'aller habiter temporairement d'autres lieux" (Knafou \& Stock, 2003) répond à la volonté de ne pas dissocier le touriste du lieu touristique. Cette conception permet d'envisager le système touristique dans son ensemble. Cependant cette définition est à compléter, compte tenu de la période étudiée, par l'apport de certains auteurs qui qualifient l'activité touristique du terme d'excursionnisme cultivé. Ce terme est éloigné de la définition d'usage mettant en avant un déplacement de loisirs en dehors de l'agglomération urbaine, sans hébergement hors du domicile (Herbin, 1983 ; OMT, 1995). Il renvoie, en ce début de XXe siècle, à "une pratique cultivée, très proche de l'activité des sociétés savantes" (Hoibian, 2000, pp.56-57). Cet excursionnisme autorise les sociétés d'encouragement, qu'elles soient montagnardes ou nautiques, à jouer un rôle d'intermédiaire culturel en permettant aux fractions intellectuelles de la bourgeoisie 
récente "de se doter des propriétés de sociabilité des milieux cultivés". En effet, les voyages sont lents et la plupart des déplacements de loisirs engendrent la découverte de nouvelles destinations et nécessitent de prévoir un hébergement. Cependant, pour des raisons de compréhension, le terme excursionnisme sera employé dans son acception actuelle prenant en compte toute personne dont la durée de séjour ne dépasse pas une journée.

2 Ainsi, la multiplication des transports et leur régularité, liée à l'homogénéisation du temps (l'horaire parisien est imposé à toute la France en 1891) permettent de développer les voyages tout en favorisant l'émergence de nouvelles temporalités urbaines. Cela offre aux citadins la possibilité de s'évader de la ville en construisant la nature et la campagne vues comme des "espaces sanitaires et comme des lieux possibles d'un loisir régénérateur et revigorant" (Csergo, 1995). Les citadins aisés sortent des villes industrieuses, polluées (Gastinel, 1894 ; Lacassagne, 1891) pour construire un espace de loisir proche de la nature offrant une parenthèse dans leur temps de citadin (Baubérot, Bourillon, 2009).

Dans un premier temps ce tourisme, issu du grand tour d'Europe, inventé par les aristocrates britanniques au XVI ${ }^{e}$ siècle, se diffuse dans l'aristocratie durant les XVII ${ }^{e}$ et XVIII ${ }^{e}$ siècle. Il permet d'achever une éducation destinée à former des hommes du monde et des hommes d'Etat (Millet, 2000). Ainsi, le tourisme se trouve essentiellement centré sur la formation des élites, de l'aristocrate et la découverte des capitales européennes (Wagner, 2007; Roche, 2003). Ce grand tour, balisé par des itinéraires urbains, se systématise et se diffuse auprès de la bourgeoisie marchande du XIX ${ }^{e}$ siècle. Il s'agit de découvrir les grandes villes européennes. Cet itinéraire de formation permet aux jeunes gens de s'initier aux voyages internationaux tout en acquérant la connaissance du vieux continent et en se familiarisant avec les diverses pratiques du commerce. Ces voyages indissociables des activités économiques se poursuivent tout en laissant une place importante à l'activité touristique mêlant curiosité et désœuvrement. Puis, avec le développement de nouveaux moyens de communication et de transport, le tourisme tend "à satisfaire, dans le loisir, un besoin culturel de la civilisation industrielle" (Boyer, 2005). Ainsi, le touriste, par son désir de s'éloigner de la ville, va, durant son temps de loisir, se rapprocher de la nature, en respirer l'air pur tout en se cultivant et en découvrant le patrimoine culturel (Drouin, 2008). Le nautisme est acteur de cette évolution en participant à la mise en tourisme des rivières de France.

4 Le Canoë Club (CC), créé en 1904, soutenu par le Touring Club de France (TCF) et le Yacht Club de France (YCF), suit cette voie. Le club est accueilli dans les locaux parisiens du TCF et du YCF lors des réunions, est invité à présenter ses activités sur leurs stands, comme par exemple, au salon automobile de 1907 ou, toujours en 1907, au salon maritime de Bordeaux. Le CC poursuit ses activités de propagande visant à développer le tourisme nautique lors de l'exposition universelle de Bruxelles en 1910 et lors de réunions nautiques organisées par le TCF et le YCF. S'adressant à un nombre restreint de sociétaires (moins d'une centaine en 1906), le CC n'en est pas moins actif. Ses membres explorent les rivières et identifient les difficultés qui entravent la navigation. Dans le même temps le TCF, fort de ses 90000 membres (Larique, 2006), crée un Comité du tourisme nautique en février $1904^{1}$ et met en place des aménagements nautiques pour permettre le développement de la navigation touristique. Il y a une réelle complémentarité de vue entre ces grandes sociétés.

5 Constitué autour de la sociabilité à l'image des Grands Cercles du XIX ${ }^{\mathrm{e}}$ siècle (Agulhon, 1977 ; Grévy, 2003), le CC recrute ses adhérents dans les classes aisées par l'entremise du 
parrainage. Issus de l'aristocratie, de la grande bourgeoisie et de la bourgeoisie (Hajek, 2007), ces canoéistes, hommes d'âge mûr, s'affirment en s'opposant aux autres types de pratiques nautiques des bords de Marne (refus de l'image des "canotiers à canotières", refus de la compétition,...) qui utilisent périssoires, podoscaphes, yoles et outriggers pour se divertir ou faire du sport en fin de semaine. Les membres du CC se tournent résolument vers le tourisme en canoë, ils pratiquent dans un premier temps dans des canoës français puis, dans un second temps, dans les canoës canadiens, plus solides et plus maniables. Ainsi, les membres du club vont favoriser une pratique liant tourisme et activité nautique.

Figure 1. Exploration de l'Eure en canoë français, 1910.

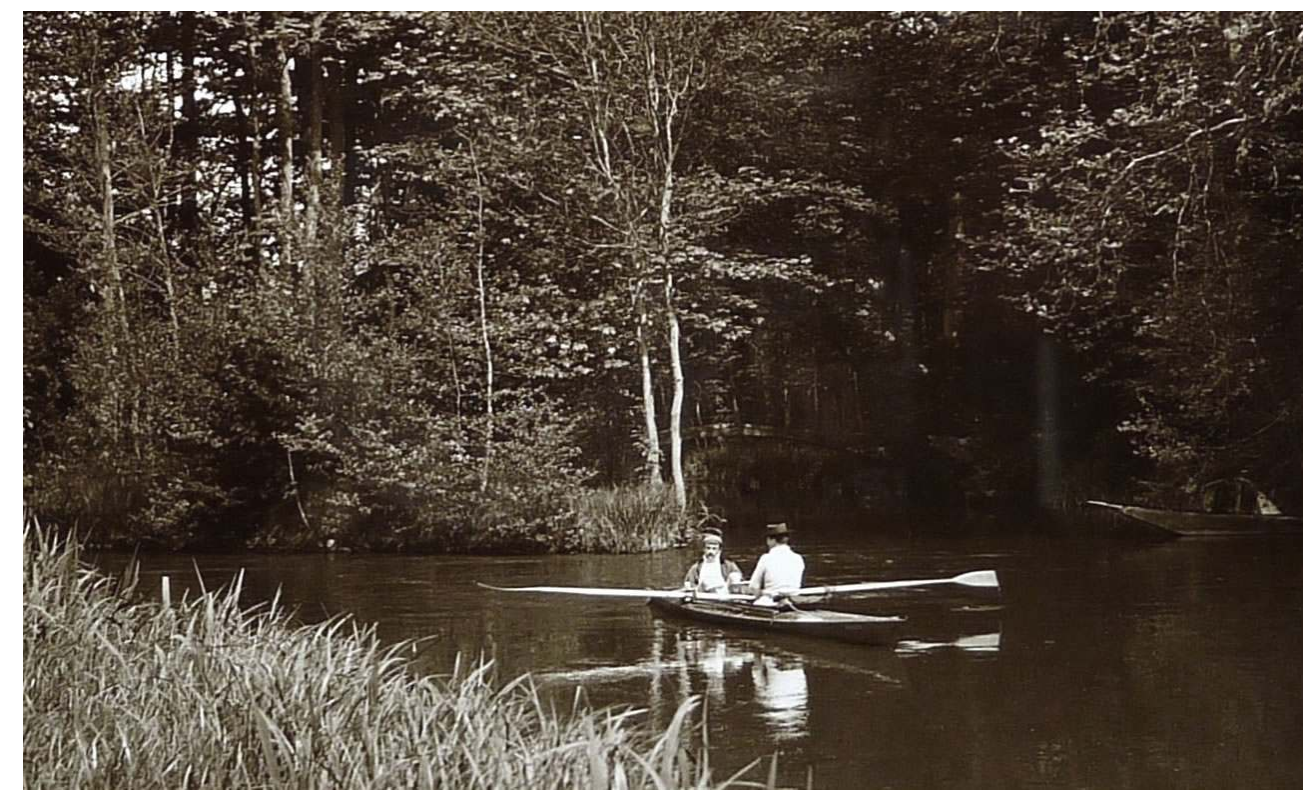

(ADC 152J22, collection Knecht)

6 Cette orientation transparait jusque dans les statuts du club qui mettent en avant l'encouragement au tourisme nautique, "mieux que cela même, une union de tous ceux qui font leurs délices du tourisme sur l'eau, afin que leur groupement devienne un jour une puissance capable de venir à bout de toutes les difficultés"2. Ainsi, très tôt les membres du CC participent à la mise en tourisme du territoire en explorant, décrivant, topographiant, photographiant les fleuves et rivières pour en permettre la navigation et développer le tourisme en canoë.

7 Les canoéistes, ouvreurs des voies (Boyer, 2002), de routes liquides, compilent des informations et les font partager aux autres adeptes des sports nautiques grâce au bulletin mensuel du CC. Ils interviennent aussi lors des conférences organisées dans les salons du YCF, du TCF, de la Société Française de Photographie et participent à la sociabilité bourgeoise parisienne. Ces "conférences avec projections sont un des plus actifs et des plus attrayants moyens de propagande" . Elles prennent la forme de causeries à partir de récits de croisières, de présentations d'excursions et d'explorations, conférences entrecoupées d'intermèdes musicaux. Ces manifestations visent à faire connaître et à encourager la diffusion de la pratique nautique dont l'expansion est favorisée par la démocratisation des moyens de transport (notamment du chemin de fer (Csergo, 2004)), les progrès de la technique et les moyens de communication (Bretin, 2002). 
8 Ainsi, les membres du CC sillonnent les rivières de France et rendent compte de leurs périples au travers de comptes-rendus dans le Bulletin mensuel du Canoë Club et de récits de voyages. Une étude du Bulletin mensuel et des récits de croisières ${ }^{4}$ permettra de mettre en lumière l'importance de cette forme de littérature de voyage dans la construction du paysage et de ses représentations, cela permettra également de comprendre la façon dont se structure le territoire, en d'autres termes la façon dont s'organise l'aménagement des bords de rivières dans le but de favoriser le développement du tourisme nautique.

9 La période choisie constitue une opportunité pour analyser le développement du tourisme nautique. En effet, le CC se met en place en 1904 pour valoriser le tourisme nautique et se positionne contre les pratiques de confrontation, pratiques sportives et compétitives mises en avant sur la Seine et la Marne par les sociétés nautiques d'aviron. Il s'agit avant tout d'un tourisme cultivé regroupant des personnes de même origine sociale et s'adonnant à la même passion pour le nautisme tout en explorant les rivières. En rendre compte à travers les récits de croisières permet au canoéiste de se positionner dans le club en tant qu'aventurier ou explorateur. Le cours d'eau devient un espace de sociabilité (Vivier, 1999) et d'aventures. Cette pratique de loisir, qui s'adresse à des gens aisés, perdure jusque dans les années 1920. En effet, en 1924, pour la première fois, le club fait paraître dans les colonnes de son bulletin une proposition de classification des rivières en fonction des difficultés et des caractéristiques physiques (pente, obstacles, largeur, débit,...5). A partir de cette date, même si la pratique du tourisme nautique perdure, elle va peu à peu être supplantée par une course à la difficulté, à l'exploit physique, prémices d'un virage sportif (Hajek, 2007).

\section{Une littérature construisant le paysage}

Le récit de croisière n'est pas destiné au simple voyageur, ne représente pas seulement "une utilité pratique ... pour les étrangers, les hommes d'affaires et les nombreux promeneurs qui aiment à parcourir et à étudier les beaux sites". Il ne s'agit pas seulement de montrer, de façon exhaustive, au voyageur les lieux de visite et la manière d'y vivre (Vajda, 2006). Le récit de croisière reste une production particulière prenant la forme d'une correspondance, d'un journal ou d'un mémoire qui, même s'il découpe l'exploration des rivières en étapes et consacre une partie du propos à l'hébergement, la restauration et la description des monuments, permet aux auteurs de rendre compte de leur implication dans le voyage. Les auteurs font partager leurs sentiments, leur vision du voyage, leur façon d'avancer au milieu des paysages, descriptions rarement neutres qui mettent en avant une "mythologie sauvage".

11 La définition du récit de croisière transparaît dans plusieurs articles des bulletins du CC parus entre juin 1906 et avril 1911. Il s'agit, d'une part, de définir les normes que doit prendre un récit de croisières et d'autre part donner les modalités d'évaluation des récits déposés. En effet, ces récits de croisières sont primés : un concours de récits de croisières est organisé chaque année par le CC. Cette action est soutenue par le TCF et le YCF qui offrent des médailles aux meilleures productions. Le premier prix est d'importance puisque s'agit, par exemple en 1906, d'un canoë offert par Albert Glandaz', fondateur du $\mathrm{CC}$, greffier en chef au tribunal de Paris, mais aussi et surtout propagateur des sports nautiques. Il soutient financièrement le club et les sports nautiques en offrant des prix mais aussi en essayant de lancer, comme c'est le cas en janvier 1909, la revue du tourisme nautique. 

qui veulent y prendre part et qui ne possèdent pas d'embarcations personnelles, peuvent emprunter, pour une période ne pouvant dépasser un mois, les trois canoës mis à leur disposition ${ }^{8}$. Le règlement impose aux candidats d'exécuter une croisière sur des rivières, lacs, canaux, côtes de France ou à l'étranger. Ils doivent faire viser une feuille de route à chaque étape par les représentants nautiques (clubs, sociétés ou membres du TCF). Ils remettent à leur retour "un récit documenté, si possible illustré".

Ainsi, parallèlement et complémentairement au récit, se développe une autre façon de rendre compte du voyage : l'illustration par la photographie. Dès 1904, la photographie et les cartes postales agrémentent les récits. Rapidement, elle va avoir toute sa place dans les récits de croisières pour en marquer de façon très précise les temps forts. Les canoéistes sont équipés d'appareils photographiques tels que le Vérascope Richard avec retardateur, le Glyphoscope (1904) permettant la photographie stéréoscopique sur plaques de verre ou simplement un Kodak. Ils gardent, ainsi, des traces de leur passage qui témoignent de la véracité de leur expédition.

Figure 2. Photographie prise avec un Glyphoscope Richard sur la Sèvre nantaise 1911.

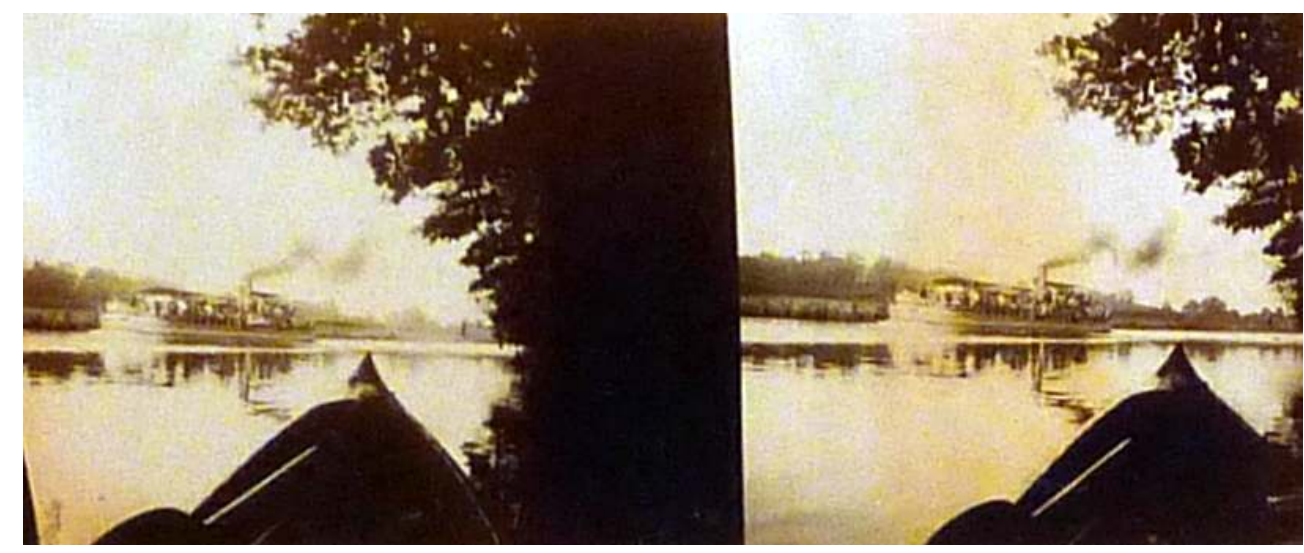

(ADC 152J25)

La photographie devient le témoin de l'expédition mais aussi le reflet de l'aménagement des fleuves et rivières et fixe sur le verre les curiosités touristiques à visiter.

Ainsi, le récit répond à une forme particulière de littérature comme l'indique un article d'Emile Dacier qui parait dans le Bulletin mensuel du CC en août 1907. Il s'intitule "Qu'estce qu'un récit de croisière ?" et précise que "ce n'est pas tout de savoir ramer, il faut encore savoir voir". L'auteur insiste pour que le récit soit le résumé des impressions "du voyage fait en bateau" et doit comprendre aussi les "impressions ressenties hors du bateaux c'est-à-dire terrestres". Il ne s'agit pas uniquement de dire "qu'on s'est levé tôt, qu'on a mis [forcé] comme un sauvage, qu'on a bu sec et mangé dru", mais bien d'attiser le désir des autres membres du CC pour qu'ils naviguent sur la rivière explorée dont le premier aura relevé les attraits.

16 Pour rendre l'information plus objective, des règles sont établies pour les récits et comptes rendus de voyage. Yasmine Marcil (2007) montre qu'un certain consensus s'établit dans cette littérature autour de trois normes: travail critique, volonté d'information du lecteur et évaluation du récit proposé par les voyageurs. Ces orientations se retrouvent dans les conseils donnés aux membres du CC désirant participer au concours de récits de croisière. Ainsi, les explorateurs nautiques sont tenus 
de multiplier les sources d'informations et de collecter les renseignements pratiques permettant d'organiser le voyage. Ces données permettent aux touristes de s'arrêter dans les lieux remarquables, de visiter les monuments et de contempler les paysages dignes d'intérêt. Enfin, le récit de croisière est aussi "un guide d'un genre spécial" dans lequel sont consignés les bons hôtels et les bonnes tables. En fait, Emile Dacier demande aux canoéistes de sortir de leur embarcation, de s'écarter de la rivière pour aller, à pied, dans les villages, visiter les églises, les châteaux pour en rendre compte. Il s'agit donc de voir, de visiter et non simplement se référer au guide Joanne communément utilisé par les touristes nautiques. Ainsi, répondre aux critères d'un récit de croisière permet d'éviter les images inexactes, tronquées et de voir les objets avec trop de rapidité (Montègne, 2009). Il y a donc une certaine fascination mais aussi méfiance vis-à-vis des descriptions de voyage qui ne rendrent pas toujours compte de la réalité.

L'inscription du récit de croisière dans ce cadre va avoir une incidence sur la construction des paysages considérés comme un espace, un territoire et la représentation qu'il en est fait. Comme l'indique Sophie Bonin (2001) la reconnaissance d'un territoire comme paysage n'est qu'une facette de l'ensemble des représentions du territoire, facette qui a l'avantage d'être conçue et perçue comme collective, partageable au sein de la société.

Les concours de récits de croisière participent à cette construction, à cette diffusion de la représentation. Les modalités de sélection mises en place dans les concours de récits de croisière renforcent encore cette orientation. Le jury, composé de membres de sociétés nautiques ou touristiques, fait partie du réseau relationnel du CC (TCF, YCF, Fédération des rameurs indépendants...). Le TCF est surreprésenté à travers son comité nautique et l'association des amateurs photographes ${ }^{9}$. Il joue, dans ce cadre, son rôle qui est de stimuler et valoriser des actions en faveur du développement du tourisme.

Les critères d'évaluation des productions littéraires marquent aussi l'importance donnée à la construction du paysage par les explorateurs nautiques :

- Point de vue de la navigation et du tourisme, les renseignements et observations recueillis (maximum : 100 points)

- Importance du voyage, considérée au point de vue de la distance parcourue et de la difficulté du parcours (maximum : 50 points)

- Valeur littéraire du récit (maximum : 50 points)

- Illustration (maximum : 50 points)

- Présentation générale du rapport (maximum : 50 points) ${ }^{10}$.

Au regard de ces critères il apparaît rapidement que le voyage et la difficulté du parcours sont minorés par rapport à sa narration. Il s'agit bien de mettre une aventure en littérature : la réalisation de l'exploration reste le support d'une production de récit. L'objectif est double, il s'agit d'abord de permettre la reproduction du parcours en donnant des informations sur la navigation et, aussi et surtout, sur les modalités de réalisation du voyage. D'autre part les récits doivent, comme le dit P-V. Stock dans un article du Bulletin de CC intitulé "Sur un récit de croisière en canoë"11, constituer de véritables joyaux littéraires, des chefs-d'œuvre d'expression, d'images originales, de bonhomie, d'humour, mettant en avant une "charmante et fine observation". Il s'agit bien au-delà du respect des modalités de concours, de mettre en littérature le paysage, de le construire. C'est un réel travail sur les représentations, les discours, la place des paysages dans la construction du monde (Claval, 2003). 


\section{Représentations et mythification du milieu naturel}

21

“Tout voyageur s'engage concrètement avec son corps dans une série de trajectoires qui sillonnent un espace donné: il traverse une certaine région en se livrant à des observations aussi bien tout au long de sa marche que dans certains points éminents où il s'arrête. La relation entre les parcours matériellement effectués par le voyageur et les parcours que celui-ci décrit dans son récit n'est (forcément) pas directe. En d'autres termes, la mise en intrigue des déambulations du voyageur résulte d'une réélaboration inventive des itinéraires effectivement accomplis lors du voyage" (Stopani, 2009). Ainsi, le voyage nautique participe à une représentation du milieu naturel. Les explorateurs nautiques réinventent les contrées qu'ils explorent pour magnifier leur randonnée et la rendre extraordinaire au regard de l'espace urbain qu'ils viennent de quitter. La rivière devient à la fois support de pratique (Clary, 1976) et lieu de rupture utopique par rapport à l'univers urbain (Bourdeau, 2007). Il y a une véritable construction touristique des rivières et des rives autour de valeurs associées aux sites, aux paysages, à l'esthétisme et la patrimonialisation (Equipe MIT, 2011, pp. 212-215).

en fleuves et les rivières en ce début de $\mathrm{XX}^{\mathrm{e}}$ siècle sont pollués, souillés par les égouts en aval des villes, parfois ils sont laissés à l'abandon depuis que le rail a supplanté le transport fluvial (Kunz, 1992), les berges et prairies se transforment avec l'industrialisation qui, comme le montre Noëlle Gerome (1996) dans son étude sur la SNECMA (Société Nationale d'Etude et de Construction de Moteurs d'Avions) et Gennevilliers, devient un obstacle à la navigation. Or, même si ces faits sont parfois relevés dans les récits de voyage, les canoéistes, dans leur grande majorité, présentent d'une façon idyllique et romantique le monde qui les entoure. Par exemple, la pollution des eaux est minorée par rapport à la description des paysages. Les récits de croisières mentionnent les inconvénients liés à la pollution comme les rejets des papeteries ou des entreprises de rouissage ${ }^{12}$, mais, dans cette première partie de siècle, les canoéistes mettent surtout en avant la nécessité d'aménager les rivières pour les rendre accessibles aux touristes nautiques.

Cela transparaît lors des préparatifs au voyage qui ressemblent assez à ceux d'une expédition dans les colonies ${ }^{13}$. Les informations rassemblées avant le départ ne sont pas fiables, les cartes et le Bottin ne sont pas à jour. Ainsi, les canoéistes, dans des canoës lourdement chargés, sont confrontés à des obstacles imprévus qui retardent leur progression sur la rivière. Equipés pour être autonomes lorsqu'il faut pallier le manque d'hébergement, les explorateurs entassent le matériel au fond du bateau : les conserves et les ustensiles de cuisine rejoignent le sachet de braises du boulanger (rapidement remplacé par le fameux réchaud à pétrole Primus, réchaud des navigateurs des années 1900) au milieu de la tente, du matelas pneumatique et du chariot pliable qui permet de rouler le canoë sur les chemins empierrés lorsque les passages sont infranchissables. A cela il faut ajouter le matériel photographique et le nécessaire à la prise de notes. 
Figure 3. Le canoë est lourdement chargé, Ain 1913.

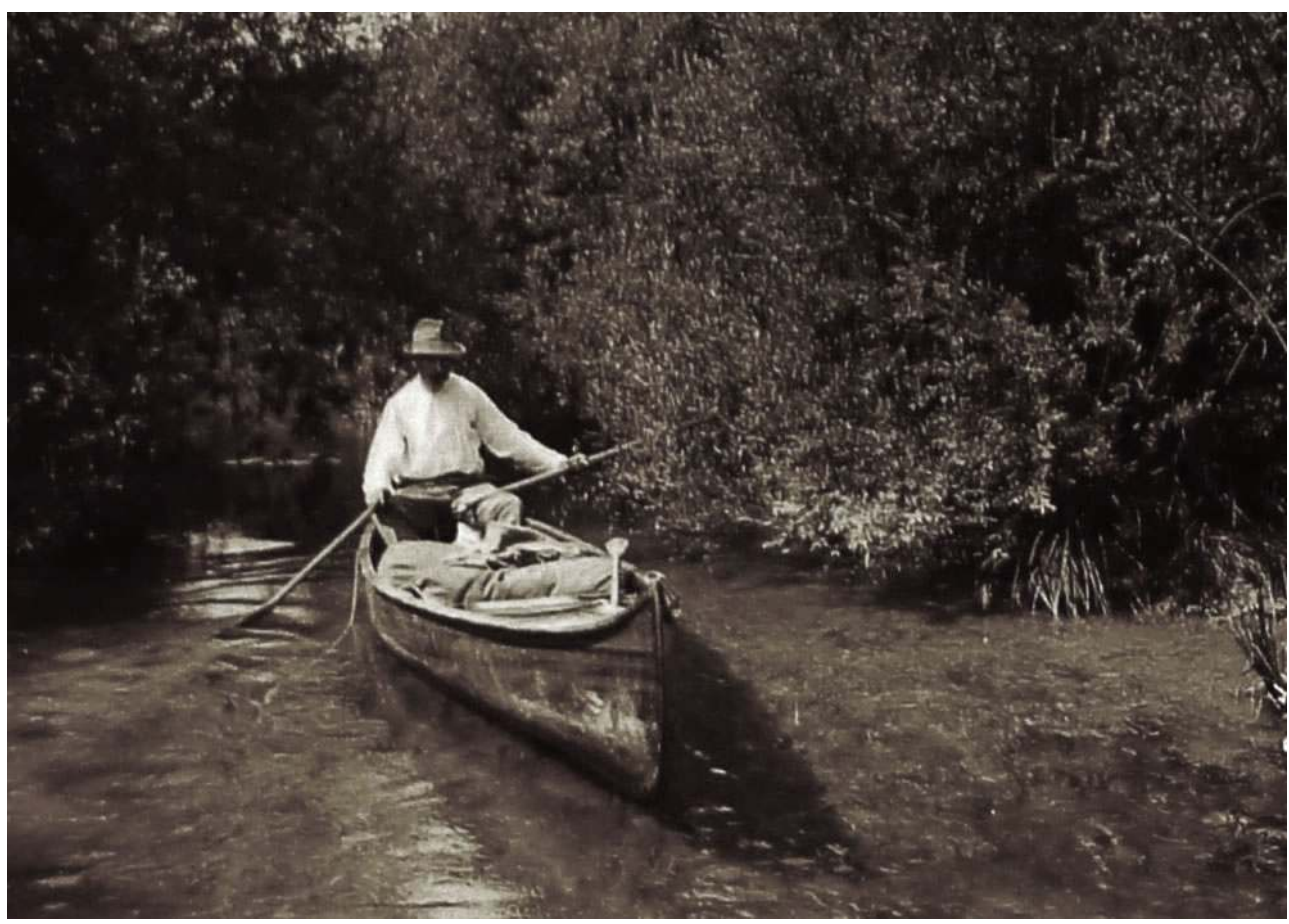

(ADC 152J25, collection Monneret) compiler des informations et les faire partager. Cependant cela reste un mythe entretenu par les membres du club qui, malgré les difficultés inhérentes à l'exploration des rivières de France, ne se séparent pas de leur costume de nage [de navigation] lorsqu'il faut parcourir le ruban d'eau mais le troquent rapidement contre des habits de ville aux étapes gastronomiques et lorsqu'il faut, le soir venu, trouver un hébergement. En fait, cette exploration nautique relève d'une part d'une certaine urbaphobie, une condamnation de la ville (Marchand, 2007; Baubérot \& Bourillon, 2009), qui pousse les classes aisées à sortir de la ville pour retrouver la nature et d'autre part d'une volonté d'imposer les codes urbains au monde rural. En effet, alors que la nature et la campagne sont encensées, la vie au grand air montrée comme source de régénération, les touristes nautiques participent, à travers leurs récits, à la sélection des hôtels et restaurants permettant de retrouver aussi le confort de la vie citadine.

Au-delà du mythe de l'exploration, la rivière, le canoë, les villages font aussi l'objet d'un autre mythe qui vise à les personnifier, à en faire des personnages imaginaires. Cette personnification renforce la notion du danger, de la difficulté. Ainsi, le ravin devient "effroyable, noir et profond", les gorges "inaccessibles", le village "malheureux"14 parce que construit au bord d'un précipice. Ces attributs vont même jusqu'à humaniser les éléments du voyage : dans les rapides on retient le canoë avec une corde comme on le ferait d'un chien attaché à une laisse ${ }^{15}$, de même le passage difficile d'un rapide devient un combat contre un ennemi implacable, la rivière. 
Figure 4. Passage de rapide en canoë canadien, Noisiel 1910.

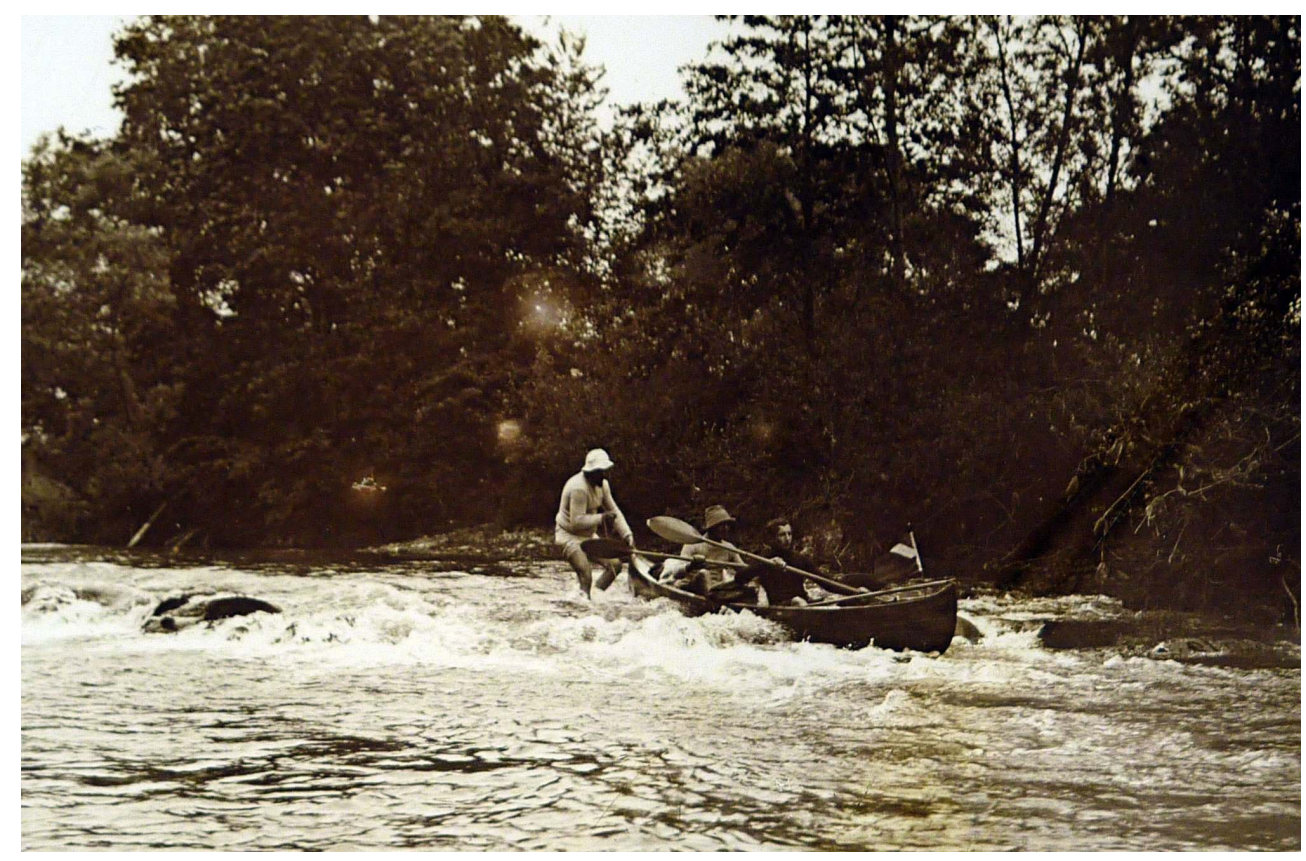

(ADC 152J21)

“Avant de s'engager dans la cascade un conseil de guerre est tenu à bord. Des félicitations sont adressées à l'ennemi loyal qui se dresse contre nous sans fard, à visage découvert. On va piquer droit sur lui par un étroit chenal vierge de rochers, on le contournera sur la droite, d'un brusque détour et puis on se rejettera dans son axe pour continuer. On part, le canoë danse dans les remous, l'écueil est à cinq mètres à peine, on va virer pour le raser sur la droite. Brusquement, au lieu d'effectuer la manœuvre convenue, l'équipier avant rejette sa pagaie à droite et "en met"16 désespérément. Son compagnon a compris, il y a trahison, un écueil invisible obstrue la passe à droite, Il faut détourner le canoë sur la gauche, cela dans une cascade, avec l'étrave déjà sur l'écueil"17. Dans ce cas, la rivière est considérée comme hostile et l'homme doit se battre pour vaincre les difficultés. Ainsi, le cours d'eau devient vivant, est humanisé. Le lyrisme des membres du CC met en avant le danger, et le caractère imprévisible, furieux ${ }^{18}$, colérique ${ }^{19}$ de la rivière. Il y a une force qui s'empare des éléments et qui impose aux canoéistes de respecter la nature. Le canoéiste se mesure au divin, aux forces naturelles (Conchon, 1997) et en ce sens ses aventures nautiques deviennent des exploits.

Dans le même temps, le récit de croisière met en avant des valeurs plus paisibles. La rivière tranquillise l'explorateur nautique citadin, lui permet de retrouver la quiétude qu'il a perdue dans les bruits de la ville industrielle et dans l'accélération du temps de ce début du $\mathrm{XX}^{\mathrm{e}}$ siècle. La croisière fluviale devient aussi, pour le canoéiste, un voyage intérieur pendant lequel la lecture du passé s'effectue au regard de la sensibilité du présent (Amirou, 2000 ; Hartog, 2003 ; Equipe MIT, 2011). Alors que le culte de la vitesse amène le voyageur à perdre le goût d'une visite approfondie des contrées (Schéou, 2007), le tourisme nautique, comme le tourisme ferroviaire à petite vitesse de la fin du XIX siècle (Blancheton, Marchi, 2011), permet, de par le rythme lent du déplacement, la découverte du paysage, le contact avec la vie des rivières. Pour ces touristes, le cours d'eau devient volupté, grâce et fraîcheur ${ }^{20}$. La promenade enchante les sens, apaise. En 
parcourant le fleuve, le navigateur longe les rives verdoyantes qui renferment le secret de la sérénité. La descente du cours d'eau est empreinte de romantisme et devient synonyme de rêverie et de sensibilité. Cette vision relève des parcours effectués par les canoéistes sur les portions calmes des rivières. Ces descriptions permettent de renforcer, par opposition, la dangerosité des parties avals du cours d'eau ou d'accentuer la palingénésie de l'homme sortant de la ville trépidante.

Ainsi, le paysage se construit autour du mythe du retour à une nature sauvage, hostile à l'homme, nature qu'il faut dompter. Cette nature influe sur la lecture des paysages qui deviennent sombres, inquiétants et dangereux mettant en avant la force des éléments et l'angulosité des reliefs. Parallèlement, une autre approche se développe, liée à la croisière nautique, dans laquelle le relief disparaît, les plaines verdoyantes et ensoleillées s'installent. Les villages et le petit peuple des fleuves s'activent. En fait, la vie autour des rivières est décrite en faisant référence aux industries et petites manufactures installées le long des berges laissant transparaître la nostalgie du passé, d'un passé respectant le rythme de la nature.

\section{Aménagement des rivières pour en permettre l'exploration}

29 Avant 1914 les rivières sont souvent mal entretenues et encombrées par des obstacles de tout ordre limitant et entravant la navigation. Le TCF va accompagner, à travers son Comité nautique, le développement du tourisme nautique en militant pour la suppression de la règlementation "imparfaite, fantaisiste et arbitraire" ${ }^{21}$ qui limite le développement du tourisme nautique. En fait, le comité nautique du TCF se donne pour programme d'aménager les rivières et les ports pour assurer la continuité de la rivière et permettre les haltes. Il œuvre pour la sécurisation des rivières (Levet-Labry, 2011) en repérant et balisant les dangers auxquels peuvent être confrontés les touristes nautiques. Son action va même plus loin puisque, loin de se limiter aux explorations en canoës manœuvrés à la rame, le comité nautique du TCF souhaite développer le tourisme nautique à moteur. Ainsi, il mène une campagne permettant de déployer sur le territoire des centres d'approvisionnement en carburant pour les yachts à moteurs. Dans la même logique, le TCF souhaite identifier et favoriser le développement des structures hôtelières permettant l'accueil des touristes aux étapes. La volonté d'Albert Glandaz, vice président du YCF et membre fondateur du comité nautique du TCF, est d'ouvrir de nouveaux espaces de pratique : "L'excursion en rivière, si elle était pratiquée davantage, ouvrirait à notre activité un champ nouveau infiniment plus varié [que l'entraînement qu'effectue le canotier aux points habituels de rassemblement]. Nos rivières, nos canaux, pénètrent partout, en toutes contrées comme les veines en l'organisme"22. Pour cela, le comité nautique du TCF va mettre en œuvre une politique d'aménagement dont le CC sera le maitre d'œuvre. Et c'est encore Albert Glandaz, fondateur du CC, qui donne la mesure de l'exploration des rivières françaises puisque dès 1904 il envisage la couverture du territoire à partir de la région parisienne. Il considère que les récits nautiques doivent donner des informations sur les parcours et participer ainsi à la diffusion de l'activité en étendant les explorations "vers les rivières plus excentriques". Ces explorations nautiques doivent amener les membres du CC à identifier les dangers et les difficultés des parcours et doivent aussi être une source d'informations sur le développement socioéconomique du territoire français. Il répond en cela au TCF qui ne cache pas ses 
ambitions lorsqu'il fixe les objectifs du tourisme nautique : "On s'instruit en voyageant. C'est notre France avec ses cultures, ses productions, ses industries, ses ressources qui défilent sous nos yeux. Tout un cours de géographie économique... au cours de l'eau". En effet, les transformations du paysage apparaissent à travers les récits de croisières, transformations qui s'inscrivent dans la modification des représentations du monde moderne.

Ainsi, on voit s'établir une complémentarité entre les explorations de rivières effectuées par le CC et leur mise en tourisme par le TCF. Lorsque les membres du CC identifient une difficulté ou un danger, une demande est faite au TCF qui essaie d'y remédier en faisant disparaître tous les obstacles sur "les routes liquides"23. Les canoéistes repèrent les parcours et rendent compte des difficultés. Les membres du CC proposent des aménagements que le TCF finance, comme par exemple en 1906 : "Le Comité du tourisme nautique du T. C. F. a décidé, sur la demande de notre vice-président M. G. Colle, de faire poser immédiatement un disque rouge, près de l'île Saint-Martin, à Pontoise, à proximité du déversoir de l'Oise. Les canotiers sauront gré au TCF de cette mesure, qui leur signalera le danger de ce déversoir que rien n'indiquait jusqu'à présent" ${ }^{24}$. Le bulletin du $\mathrm{CC}$ est le témoin de ce travail commun. Les dangers sont marqués, ils concernent les piles de pont immergées, les barrages et déversoirs ${ }^{25}$. Des aménagements sont effectués. Des pontons d'accostage sont créés ${ }^{26}$ pour permettre aux touristes nautiques de débarquer en toute sécurité près des villages et profiter des hébergements ou tout simplement sortir les canoës de l'eau avant d'effectuer un portage nécessaire pour éviter un passage dangereux. Le TCF équipe aussi les barrages de "passe canots" ${ }^{27}$ permettant d'installer des chariots sur rail pour transporter les lourds canoës chargés de leur équipement. Enfin des poteaux indicateurs sont implantés ${ }^{28}$ sur les berges pour faciliter la navigation.

31 Ces aménagements sont nécessaires car les croisières s'effectuent avec des canots pontés, dotés d'un siège à coulisses. Ils sont rapides mais fragiles, lourds et difficilement manœuvrables. Il est difficile de passer les rapides, les barrages et de hisser les embarcations sur les berges empierrées. Les embarcations lourdement chargées accentuent les difficultés de la rivière: les portages sont malaisés et deviennent vite pénibles. Il faut, lorsque la difficulté est trop importante, débarquer, décharger le bateau pour le recharger quelques centaines de mètres plus loin. Il est donc important dans les premières années du club de participer avec le TCF à l'aménagement des rivières. Ainsi, les descriptions des installations favorisant le développement du tourisme nautique sont prégnantes dans les récits de croisière jusqu'en 1911. Les récits mettent aussi en avant les difficultés, les embarcadères, les écluses. L'éclusage est un élément important du voyage. Il permet de mener à bien le voyage mais est soumis au bon vouloir des éclusiers ou de l'ingénieur des Ponts et Chaussées qui peuvent, parfois, abuser de leur autorité et retarder l'avancée de l'expédition.

Pour parfaire le relevé des informations nécessaires à la reproduction de la descente de rivière, hôtels et garages à bateaux doivent être identifiés pour construire l'itinéraire. Les étapes sont calculées pour offrir un temps de repos aux canoéistes fourbus par une descente de rivière parfois longue et difficile. Elles permettent également quelques visites de sites remarquables (châteaux, églises, points de vue...). Les haltes se situent dans des villes de moyenne importance pouvant mettre à disposition hébergement et lieu de stockage pour l'embarcation. Elles servent aussi à tisser des liens avec d'autres sociétés nautiques et avec les délégués du TCF qui signent la feuille de route. Elles permettent de passer des accords avec les hôteliers pour obtenir des conditions favorables 
d'hébergement comme c'est le cas lors de la croisière Contamin sur la Marne, l'Aisne et l'Oise en 1906: "Après diner, le propriétaire de l'hôtel signe un accord avec le CC pour le garage et demande même son admission dans la société. Tous ceux de nos camarades qui passeront à Toutevoye feront bien de s'y arrêter, ils y trouveront bon gîte et bon accueil pour eux et leur embarcation" ${ }^{29}$. Faisant suite à cela, dès août 1906, le bulletin du CC établi la liste des garages à bateaux recommandés qui permettent de laisser en sécurité les canoës tout en ayant un accueil convenable. Ils se situent parfois, comme nous l'avons vu, dans des hôtels, à l'exemple de celui de M. Fétillieux à Saint-Adrien près de Rouen (Seineinférieure) qui offre gratuitement le garage si le canoéiste descend dans son hôtel. Il semble qu'il existe une corrélation importante entre la diffusion par le CC du canoë sur le territoire et l'existence de lieux d'accueil, qu'il s'agisse des garages ou des hébergements. A travers les récits de croisière il devient possible de découvrir l'émergence d'une organisation touristique le long des rivières avec, comme points de repères, les étapes journalières. L'étape, le temps de navigation et l'écluse deviennent des éléments essentiels du voyage. Des hôtels, restaurants et garages à bateaux sont référencés et participent au maillage touristique déjà engagé, quelques années auparavant, par le TCF avec la bicyclette et l'automobile.

L'arrivée du canoë canadien, d'abord importé puis construit en France, va transformer la pratique et par voie de conséquence la représentation de la difficulté et des nécessités d'aménagement. Moins rapide mais plus léger et robuste il permet d'étendre le rayon d'action des canoéistes. Les accostages peuvent se faire sans que des aménagements particuliers soient nécessaires. Sa maniabilité permet d'explorer le cours supérieur des rivières, parties plus étroites et plus tourmentées. Les récits de croisières rendent compte de cette transformation. Le camping, hébergement léger, se développe car il permet une certaine autonomie aux explorateurs. Depuis 1905, le TCF essaie de mettre à la mode l'hébergement sous toile. Le camping est mis en avant par le CC lorsqu'il s'agit de promouvoir le canoë canadien ${ }^{30}$. Cependant sa diffusion est lente. En février 1907 la première publicité parait dans le bulletin du $\mathrm{CC}$ et la première descente utilisant le camping "à grande échelle" est relatée en décembre 1907 dans les pages du Bulletin du CC. Le baron Henri de Lormais en compagnie de Jean Lintz, à bord du canoë Titi, réalisent la descente de la Seine de Courbevoie à La Bouille en ne dormant que deux nuits sur les six à l'hôtel. Le camping a été le mode d'hébergement privilégié par cette expédition. Cependant, il faut attendre les années 1912 pour voir ce type d'hébergement ${ }^{31}$ apparaître réellement dans les récits de croisières. A cette période un débat s'installe dans le Bulletin du CC sur la nécessité et l'utilité du camping. Ce débat va opposer partisans et détracteurs du camping jusqu'en 1924. Malgré les réticences, l'élan est donné et de nombreuses croisières s'effectuent avec ce mode d'hébergement, canoë et camping semblant faire cause commune dans la découverte de l'activité de plein air ${ }^{32}$. L'hébergement en camping met en avant la quête du pittoresque et de l'aventure, prend une place importante dans la découverte de nouveaux territoires de jeu (Sirost, 2001, 2002) et permet un accès plus aisé au bassin supérieur des rivières. Dans l'après-guerre, il devient une alternative qui permet de réduire sensiblement les coûts de logement des expéditions nautiques. L'arrivée dans les villages avec un canoë constitue une aubaine et une attraction à laquelle tous les villageois veulent assister ${ }^{33}$. Les canoéistes dynamisent le commerce local pour refaire leurs vivres : aubergistes et meuniers, fermiers se mobilisent pour fournir pain, œufs et vin aux randonneurs nautiques. Cependant les réactions sont différentes lorsque les canoéistes s'installent pour camper. Objets de curiosité (Tissandier, 2001), parfois considérés comme des "Romanichels" ${ }^{34}$, des voleurs ou des espions ${ }^{35}$, ils sont 
régulièrement contrôlés par la maréchaussée à la demande des habitants. Néanmoins, le lien entre le canoë et le camping, ténu au début, devient plus solide. Il va donner la possibilité d'ouvrir des parcours dans les hautes rivières, provoquant une inflexion de l'activité nautique vers une pratique plus sportive.

Ces transformations dans la pratique marquent les récits. Dans un premier temps, il est fait éloge de la modernisation, du développement des industries et manufactures sur les bords de rivières. Le modernisme est mis en avant avec les constructions de centrales hydrauliques. Le cours d'eau est présenté comme producteur d'activités industrielles et de richesses ${ }^{36}$. Le récit permet de repérer le développement de l'activité humaine le long des rives. En ce sens il est la marque de la transformation du paysage et de la société humaine. Le passage à la société moderne se lit à travers le développement des industries liées à la houille blanche et à l'abandon des activités traditionnelles de la rivière.

La Première Guerre Mondiale va transformer le paysage. Certaines infrastructures anciennes sont détruites pour rendre les voies navigables et normaliser les canaux. C'est le cas de certains ponts anciens dont l'arche était trop basse pour permettre le passage de convois fluviaux ${ }^{37}$. De même, progressivement les moulins et autres constructions liées aux activités rurales disparaissent au profit de barrages industriels et d'usines. Les constructions modernes transforment la rivière, la canalisent, l'endiguent pour prévenir les inondations. Les récits notent ces transformations. Les constructions et bâtiments anciens sont mis en avant dans les descriptifs comme des éléments du paysage, des points de repères et non plus comme difficultés. Les usines modernes ne sont plus considérées comme les vecteurs d'entrée dans le monde moderne mais plutôt comme des verrues dans le paysage ("elles dressent leurs menaçantes carcasses"38). Les descriptions se tournent vers les villages traditionnels. Il existe une réelle nostalgie de la période heureuse d'avant 1914 où la rivière était encore à redécouvrir. Dans le même temps, la pratique du canoë se transforme et s'oriente vers l'exploration des hautes rivières comme s'il s'agissait de rester proche d'une nature sauvage plus difficile d'accès à la modernité. Les basses rivières sont explorées, les conditions de pratique évoluent et permettent d'ouvrir l'accès aux hautes rivières. Alors que la pratique se développe et se démocratise, les premiers guides à usage exclusif des canoéistes paraissent à l'initiative du TCF et avec l'aide du CC. Ils vont avoir un format plus pratique, plus facile à transporter lors des explorations nautiques. Ils vont aussi devenir plus précis, permettant le développement d'une pratique plus sportive initiée entre autres par le classement des rivières effectué en 1924 par le CC en fonction des difficultés de navigation. Les récits perdent leurs caractéristiques initiales et deviennent, peu à peu, des guides techniques focalisés sur les difficultés liées à la pratique sportive.

\section{Conclusion}

Le récit de croisière a été, pour le Canoë Club, un des moyens utilisés pour développer, avec l'aide du Touring Club de France, le tourisme nautique sur le territoire. Il apparait rapidement, notamment à travers les concours de récits de croisière, que la narration de l'aventure prend plus d'importance que le voyage et ses difficultés objectives. Il s'agit de mettre en avant les attraits d'un parcours et de rendre le voyage proche des représentations de la nature que se font les citadins. Ainsi, le travail sur le mythe d'une nature sauvage à explorer construit le paysage en rendant l'exploration nautique aventureuse au regard de l'espace urbain occupé habituellement. La randonnée nautique 
devient un exploit physique mais aussi un voyage intérieur pendant lequel le canoéiste se confronte avec le passé et son passé. L'inconnu devient source de curiosité et d'exploit. Cet exploit est construit, comme le récit. En effet, un travail important est effectué pour aménager physiquement les rivières, en faciliter l'exploration et permettre une reproduction des parcours. Ainsi, d'une part le récit de croisière met en avant le mythe mais aussi participe à la construction d'une mise en sécurité des descentes.

La classification des rivières en fonction du niveau des difficultés en 1924 met un frein à cette pratique nautique touristique. Les récits de croisières changent et deviennent des guides plus techniques dans lesquels le paysage disparaît peu à peu au profit d'une description des difficultés techniques. Cette évolution vers la pratique de la descente des hautes rivières va de pair avec le développement de l'activité sportive. L'activité se transforme, la confrontation entre les canoéistes et la nature devient la règle. Il s'agit de vaincre les difficultés et non plus parcourir les rivières pour l'attrait des paysages. La sportivisation de la pratique du canoë marque la seconde partie du XX siècle et débouche sur une multiplication des activités avec la mise en avant du risque. L'homme passe d'une confrontation à l'inconnu à une confrontation avec la mort.

Les pratiques à risques se diversifient (nage en eau vive, raft, hautes rivières, ...) repoussant sans cesse les limites du possible. Malgré cette orientation sportive, le tourisme nautique, après avoir été mis en veille pendant le dernier quart de siècle semble redevenir une pratique partagée sous le vocable du loisir nautique. Cette pratique participe à l'offre d'activités de loisirs des stations touristiques. Cette orientation se retrouve dans la diversification des clubs, notamment celle du Canoë Club de France, héritier du CC, qui aujourd'hui compte parmi ses adhérents environ $40 \%$ de participants à une activité de loisir et de détente.

\section{BIBLIOGRAPHIE}

AGULHON M. (1977), Le cercle dans la France bourgeoise 1810-1848. Etude d'une mutation de sociabilité, Armand Colin.

AMIROU R. (2000), Imaginaire du tourisme culturel, Presses Universitaires de France.

BAUBEROT A., BOURILLON F. (2009). Urbaphobie, la détestation de la ville aux XIX ${ }^{e}$ et XX $X^{e}$ siècles, Paris, Bière.

BERTHO-LAVENIR C. (1999), La roue et le stylo, Comment sommes-nous devenus touristes ?, Odile Jacob.

BLANCHETON B., MARCHI J.-J. (2011), “Le développement du tourisme ferroviaire en France depuis 1870", Histoire, économie \& société, 3, 30ª année, pp. 95-113.

BONIN S. (2001), "Paysages et représentations dans les guides touristiques : La Loire dans la collection des Guides Joanne, Guides Bleus (1856 à nos jours)", L'Espace géographique, 30, 2, pp. 111-126. 
BOURDEAU P. (2007), "Les sports de nature comme médiateurs de l'entre-deux ville -montagne", in MONTEVENDI-WEBER L., DESCHENEAUX C., TRANDA-PITTION M., Campagne-ville, le pas de deux, Lausanne, Presses polytechniques et universitaires romandes, pp. 27-36.

BOTTI L., PEYPOCH N., SOLONANDRASANA B. (2008), Ingénierie du tourisme. Concepts, méthodes, applications, De Boeck.

BOYER M. (2002), “Comment étudier le tourisme ?”, Ethnologie française, XXXVII, pp. 393-404.

BOYER M. (2005), Histoire générale du tourisme du XVI $I^{e}$ au XXIe siècle, Paris, Harmattan.

BRETIN K. (2002), “Avènement des sports nautiques à Châlon-sur-Saöne (1877-1960). La

modernisation de la société à la rencontre des traditions”, Sciences et Motricité, 45, pp. 67-84.

CLARY D. (1976), “Tourisme et aménagement régional”, Annales de Géographie, 85, 468, pp. 129-154.

CLAVAL P. (2003), Paris et Londres au XIXe siècle. Représentations dans les guides et récits de voyages, Paris, Hancock, CNRS Editions.

CONCHON A. (1997), "Introduction”, Hypothèses, 1, pp. 67-75.

CSERGO J. (1995), "Extension et mutation du loisir citadin, Paris XIX ${ }^{\mathrm{e}}$-début XX", in CORBIN A., L'avènement des loisirs 1850-1960, Aubier, pp. 121-167

CSERGO J. (2004), "Partie de campagne. Loisirs périurbains et représentation de la banlieue parisienne fin XVIII-XIX ${ }^{\mathrm{e}}$ siècle”, Sociétés et représentations, 17, 1, pp. 15-50.

DROUIN M. (2008), “Ouvrir les canaux à de nouveaux publics, le patrimoine comme médiateur du tourisme nautique”, Téoros, pp. 85-88.

EQUIPE MIT (2011), Tourisme 3. La révolution durable, Belin.

GASTINEL A. (1894), Les égouts de Paris, Paris, Jouve.

GEROME N. (1996), “Une emprise industrielle hégémonique : le SNECMA”, in FOURCAUT, La ville divisée, Créaphis.

GREVY J. (2003), “Les cafés républicains de Paris au début de la Troisième République. Etude de sociabilité politique", Revue d'histoire moderne et contemporaine, 50, 2, pp. 52-72.

HAJEK S. (2007), Histoire culturelle d'une société nautique : le Canoë Kayak Club de France 1904-2004, Thèse de $3^{\mathrm{e}}$ cycle, Université Paris V.

HAJEK S., MARSAC A. (2008), "Les récits de croisière : développement d'une culture touristique du canoë dans la France de l'entre-deux-guerres", Loisir et société, 31, 2, pp. 233-265.

HARTOG F. (2003), Régimes d'historicité, Seuil.

HERBIN J. (1983), “Les loisirs de proximité des habitants de trois villes alpines : Grenoble, Annecy et Chambéry", Norois, 120, pp. 597-610.

HOIBIAN O. (2000), Les alpinistes en France 1870-1950 : une histoire culturelle, L'Harmattan.

KNAFOU R., STOCK M. (2003), article "tourisme", in LEVY J., LUSSAULT M., Dictionnaire de géographie du tourisme, Belin.

KUNZ A. (1992), “Voies navigables et développement économique”, Histoire, économie et société, 11, 1, pp. 13-17.

LACASSAGNE A. (1891), L'hygiène à Lyon. Compte-rendu des travaux du Conseil d'Hygiène Publique et de Salubrité du département du Rhône, Lyon, Storck. 
LARIQUE B. (2006), L'économie du tourisme en France des années 1890 à la veille de la Seconde Guerre Mondiale. Organisation et développement d'un secteur socio-économique, Thèse de doctorat d'histoire, Université Bordeaux III.

LEVET-LABRY E. (à paraître), Aménager les rivières et réduire les risques pour développer le tourisme nautique en France (1904-1924), Vertigo.

MARCHAND B. (2007), L'urbaphobie en France depuis 200 ans : très bref résumé, Colloque "Ville Mal Aimée, Ville à Aimer", Château de Cerisy-la-Salle, 5-12 juin 2007.

MARCIL Y. (2007), “La fureur des voyages. Les récits de voyage dans la presse périodique (1750-1789)", Réseaux, 141-142, 2, pp. 378-381.

MILLET B. (2000), "Le grand tour, un paysage photographique", La pensée de midi, 3, 3, pp. 63-68.

MONTEGNE G. (2009), “Parcours romains, parcours méditerranéens : L'impact du voyage à Rome dans la construction des territoires au siècle des Lumières”, Rives méditerranéennes, 34, 3 , pp. 45-56.

OMT (1995), Concepts, définitions and classifications for tourim statistics, Technical manual $n$ 1, Madrid, World Tourisme Organisation.

ROCHE D. (2003), Humeurs vagabondes : de la circulation des hommes et de l'utilité des voyages, Paris, Fayard.

SCHEOU B. (2007), “De la tyrannie de la vitesse à l'eurythmie : le temps d'exister, Application au voyage touristique", Téoros, Automne 2007, pp. 15-24.

SIROST O. (2001), "Les débuts du camping en France : du vieux campeur au village de toile", Ethnologie française, XXXVII, 2, pp. 607-620.

SIROST O. (2002), "Se mettre à l'abri ou jouer sa vie ? Eléments d'une culture sociale du risque", Sociétés, 77, pp. 5-15.

STOPANI A. (2009), “De l'itinéraire au territoire : Pratiques et mises en représentation de l'espace”, Rives méditerranéennes, 34, 3, pp. 11-26.

TISSANDIER A. (2001), "Les débuts du camping en Auvergne”, Ethnologie française, XXXVII, 2, pp. 641-650.

VAJDA J. (2006), "Paris en huit jours : À la découverte de la ville à travers les guides, les journaux pour touristes et les récits de voyage, 1855-1937”, Sociétés \& Représentations, 21, 1, pp. 255-273.

VIVIER C. (1999), La sociabilité canotière : la société nautique de Besançon, Paris, L'Harmattan.

WAGNER A-C. (2007), "La place du voyage dans la formation des élites", Actes de la recherche en sciences sociales, 170,5 , pp. 58-65.

\section{NOTES}

1. Revue mensuelle du TCF, février 1904.

2. Bulletin mensuel du Canoë Club, avril 1906.

3. Bulletin mensuel du Canoë Club, mai-juin 1908.

4. Archives de Créteil (ADC), cote 152J.

5. Bulletin du CC, octobre 1924 et novembre 1924.

6. Chaix Napoléon, Indicateur officiel des environs de Paris, $n^{\circ} 27,1859$. 
7. Albert Glandaz est membre fondateur du Canoë Club en 1904. Il fut aussi président de la Fédération des Sociétés d'Aviron, vice-président du Yacht Club de France, membre fondateur du Comité Nautique du Touring Club de France, membre du Cercle de Voile de Paris et président du Comité National de Sports (l'un des ancêtres du Comité National Olympique et Sportif Français) entre 1910 et 1911. Il est l'un des mécènes des sports nautiques en France.

8. Bulletin mensuel du CC, juin 1906.

9. Bulletin mensuel du CC, mars 1908.

10. Bulletin mensuel du CC, mai-juin 1908.

11. Bulletin mensuel du CC, février 1907.

12. Carnet du canoéiste, Le Loir, Bulletin du CC, $\mathrm{n}^{\circ} 55$, septembre 1910. Carnet du canoéiste, Quelques rivières du Cotentin, Bulletin $d u C C, n^{\circ} 49$, mars 1910.

13. Croisière en petite Seine et haute Seine, 1909, ADC 152J24.

14. Boulade M-J., La Creuse et la Vienne en canoë de Fresselines à Chinon, Imprimerie commerciale et agricole de Compiègne, 1910, p. 29.

15. "Le canoë est tenu comme on le ferait d'un chien attaché à une laisse "(ADC 152J125 L'Ain par Monneret 1913.

16. "En met" : Le canoéiste pagaie avec force.

17. ADC 152J25 Ain, récit de croisière de Monneret 1913.

18. ADC, carton 152J26: Creuse 1913.

19. ADC, carton 152J25 : Ain 1913.

20. Bulletin mensuel du Canoë Club, août 1907.

21. Revue mensuelle du TCF, février 1904.

22. Revue mensuelle du TCF, mars 1904.

23. Bulletin mensuel du CC, avril 1906.

24. Bulletin mensuel du CC, mars 1906.

25. Bulletin mensuel du Canoë Club, juin 1907.

26. Bulletin mensuel du Canoë Club, mai 1906.

27. Revue du TCF, août 1909. ADC, carton 152J16: En Canoë, la descente de la Garonne d'Agen à Bordeaux, 1904.

28. Bulletin mensuel du Canoë Club, août 1907.

29. ADC, carton 152J23 : H. \& L. Contamin, Croisière sur la Marne, Aisne, Oise, 1906.

30. Bulletin mensuel du CC, septembre 1906.

31. Bulletin mensuel du CC, octobre 1912.

32. Bulletin mensuel du C.C., août 1912, septembre 1912, juin-juillet 1922, septembre 1922

33. ADC, Carton 152J25 : Ain 1913.

34. ADC, Carton 152J 25 : L'Ain, 1913, p 25-27.

35. En 1903, trois canoéistes allemands désirant descendre le Doubs, la Saône et le Rhône sont arrêtés par la gendarmerie et sont pris pour des espions car ils naviguaient sans permis. Bulletin mensuel du Canoë Club, juin 1906.

36. Bulletin mensuel du CC, janvier 1911.

37. Bulletin mensuel du CC, octobre 1919.

38. Ibid. 


\section{RÉSUMÉS}

Les récits de croisière constituent une littérature qui révèle le développement du tourisme nautique. La redécouverte des rivières françaises, effectuée par des citadins aisés, relève de mythes qui vont fonder l'action de ces touristes : le retour à la nature et la personnification des éléments. Il s'agit de mettre en avant l'exploit de ces citadins, qui sortent des villes, et sont confrontés à la nature et au monde rural. La confrontation entre un monde idéalisé et un territoire transparaît dans les récits. Les aménagements des rivières constituent la partie visible du rapport du citadin à la nature. L'espace à explorer doit être aménagé pour permettre au citadin de pratiquer une activité proche de l'élément naturel. Mais cet espace ne doit pas prendre les traits de la ville qu'il vient de quitter.

Travel stories constitute a literature that reveals the development of nautical tourism. The rediscovery of French rivers, carried out by well-off city dwellers, is based on myths about the return to nature and the personification of the elements. It's a matter of highlighting the feat of these townsmen, who leave the cities and face nature and rural life. The confrontation between an idealized world and a territory shows through the stories. The development of the rivers constitutes the visible part of the relationship between the city dwellers and nature. The space to explore must allow the city dwellers to go in for an activity closely related to nature. But this space should not take the features of the city he has just left.

\section{INDEX}

Keywords : travel stories, tourism, landscape, river, nature, myth

Mots-clés : récits de voyage, tourisme, paysage, rivière, nature, mythe

\section{AUTEUR}

\section{ERIC LEVET-LABRY}

Université Paris-Est, Laboratoire Analyse Comparée des Pouvoirs, E.A. 3350, 5 boulevard Descartes Champs sur Marne, 77454 Marne-la-Vallée, Eric.levet-labry@univ-mlv.fr 